\title{
Covid-19: Do vaccines work against omicron-and other questions answered
}

\author{
The SARS-CoV-2 omicron variant, first detected in South Africa on 24 November, has now been found \\ in 57 countries. Elisabeth Mahase looks at what we know about it so far, including how well \\ treatments and vaccines work
}

\section{Elisabeth Mahase}

\section{What mutations does omicron have?}

Omicron's spike protein has at least 30 amino acid substitutions, three small deletions, and one small insertion, says the US Centers for Disease Control and Prevention. The CDC has highlighted four key mutations that may aid transmission: N501Y, H655Y, $\mathrm{N} 679 \mathrm{~K}$, and $\mathrm{P} 681 \mathrm{H}$, the last of which has also been found in the alpha variant, while a different mutation at this position (P681R) is found in delta. ${ }^{1}$

\section{Is it more transmissible than the delta variant?}

Early indications are that omicron is more transmissible than previous variants, especially as it has overtaken delta as the predominant variant in South Africa, and there is evidence indicating that it comes with an increased risk of reinfection. ${ }^{2}$

The UK's pandemic modelling group SPI-M-O has warned that although data from South Africa are subject to caveats there is a strong indication that omicron has a "significant transmission advantage, significant immune escape, or both, or some other fitness advantage over the predominant variant (delta)." It said that the current evidence presents a "compelling case for omicron to cause a wave of infection in the UK." 3

England's health secretary, Sajid Javid, told Sky News on 8 December, "It spreads faster than any other covid-19 variant we've seen so far ... We estimate [it's doubling time] is between two and a half to three days, which would mean at this rate by the end of this month we could hit about a million infections in the community throughout the UK."4

\section{Does it lead to more severe disease?}

It has been suggested that although omicron is more transmissible than delta, it causes less severe disease. Occupancy of intensive care beds in South African hospitals between 14 November and 4 December was $6.3 \%$, which the World Health Organization said was "very low compared with the same period when the country was facing the peak linked to the delta variant in July."

Further data from one of the worst affected health districts in South Africa showed that, over the same time period, 98 of 1200 people admitted to hospital were receiving supplemental oxygen and four were being ventilated. "This is very preliminary data with a small sample size, and most of the people admitted to the health facilities were under the age of 40 . As the clinical profile of patients changes, the impact of omicron may change," WHO said. ${ }^{5}$

Speaking at a German Science Media Centre briefing on 8 December, Florian Krammer, professor of vaccinology at Mount Sinai Hospital in New York, said, "We can only speculate about this. We have to be very careful here, because we're talking about a young population [in South Africa] that has a very high baseline immunity from previous infection, and that's why we may be seeing a little bit of a milder disease. What we need to assume is that omicron will be very similar to the other variants in terms of severity."

Other health leaders have also warned that, even if omicron does cause less severe disease, its infection rate could have "devastating consequences." Tim Spector, professor of genetic epidemiology at King's College London, who runs the Zoe covid-19 symptoms app and study, said, "If numbers skyrocket, it doesn't matter if the percentage of people being hospitalised or dying remain low-it's about volume, not percentages. The higher rate of transmission even in the vaccinated could have devastating consequences, and hundreds of people continue to die every week as we head into our second Christmas of this pandemic." 6

\section{Does omicron escape the vaccines?}

Findings from a very small study involving just 12 people in South Africa, which have been released through a preprint, indicate that the efficacy of the Pfizer-BioNTech vaccine could be significantly reduced against omicron, with a 41-fold lower level of neutralising antibodies when compared with a variant of the virus that was widespread in the early stages of the pandemic (characterised by spike protein substitution $\mathrm{D} 614 \mathrm{G}){ }^{7}$

Jonathan Ball, professor of molecular virology at the University of Nottingham, said, "Whilst the amount of virus killing observed in the lab is reduced markedly-up to 40-times reduction-there is still measurable virus neutralisation, especially in those who were vaccinated and previously infected. This group effectively mimics what we would expect in people who have had two doses of vaccine plus a boost ... That's why we still need to get the message across: get vaccinated, get boosted, even if you have been infected before."

Pfizer said its research indicated that a third dose of vaccine provided similar levels of neutralising antibodies against omicron as seen after two doses 
against the original virus (wildtype). ${ }^{8}$ In its statement the company said that people who had had two vaccine doses exhibited "more than a 25 -fold reduction in neutralisation titers" against omicron when compared with wildtype, suggesting that two doses of the Pfizer vaccine "may not be sufficient to protect against infection with the omicron variant.” The company said "additional studies indicate that a booster with the current covid-19 vaccine increases the antibody titers by 25 -fold."

\section{Could there be an updated vaccine?}

Pfizer and BioNTech started developing an omicron specific version of their vaccine on 25 November and said that the first batches could be ready for delivery within 100 days, pending regulatory approval. Moderna is also working on an updated version against the new variant and said it could be finished with testing and ready to file with the regulators by March 2022. ${ }^{9}$

However, speaking at the German Science Media Centre briefing in relation to the Oxford-AstraZeneca vaccine, Teresa Lambe, lead scientific investigator at the University of Oxford's Jenner Institute, said, "We don't know if we need a new vaccine yet. It is very, very likely that we will see a fall in neutralising antibodies, [but] we have yet to come across a variant where we've seen an impact on protection against hospitalisation and death. Unfortunately, we need to be a little patient for that data to come out. We, like other vaccine manufacturers, can go fast. We've already made a different variant vaccine, AZD2816, for the beta variant, so we've got the processes and the willpower to go fast if we need to."

\section{What about antiviral and antibody treatments?}

GSK has said that its monoclonal antibody sotrovimab, approved in the UK last week, ${ }^{10}$ retains activity in vitro against the full known omicron spike protein. The preclinical data were generated through testing of a model of the variant that included the 37 mutations identified in the spike protein. However, the biotechnology company Regeneron has reported that early testing of its monoclonal antibody treatment Ronapreve indicated that it may have reduced efficacy against omicron. ${ }^{11}$ No information has yet been provided on the efficacy of the SARS-CoV-2 antiviral molnupiravir against omicron. ${ }^{12}$

\section{Can PCR testing detect omicron?}

Current PCR testing does detect the variant, although several laboratories have said that one widely used test doesn't detect one of the three target genes (S). This is the same gene dropout that was seen with the alpha variant in late 2020. ${ }^{13}$ WHO has said that this dropout can be used as a marker for the omicron variant, pending sequencing confirmation. ${ }^{2}$

\section{What are countries doing in response?}

Many countries have implemented travel bans or tighter restrictions on travellers from some African countries. The UK, where more than 800 cases of omicron have been detected so far, has placed 11 African countries on its red list: Angola, Botswana, Eswatini (formerly Swaziland), Lesotho, Malawi, Mozambique, Namibia, Nigeria, South Africa, Zambia, and Zimbabwe. This has raised questions regarding why African countries are facing these measures when the variant is circulating in many European countries as well. ${ }^{14}$

Alongside travel restrictions, the UK has also put some additional domestic measures in place, including recommending that people work from home where possible and wearing face masks in many indoor public places, such as cinemas and theatres. ${ }^{15}$ Some other European countries, including Austria and Germany, already had some form of regional or national lockdown in place before the variant was widely reported. It is unclear how omicron will affect the lifting of these restrictions.

1 Centers for Disease Control and Prevention. Science brief: omicron (B.1.1.529) variant. Dec 2021. https://www.cdc.gov/coronavirus/2019-ncov/science/science-briefs/scientific-brief-omicronvariant.html.

2 World Health Organization. Classification of omicron (B.1.1.529): SARS-CoV-2 variant of concern. Nov 2021. https://www.who.int/news/item/26-11-2021-classification-of-omicron-(b.1.1.529)-sarscov-2-variant-of-concern.

3 SPI-M-O. Consensus statement on covid-19.7 Dec 2021. https://www.gov.uk/government/publications/spi-m-o-consensus-statement-on-covid-19-7-december-2021/spi-m-o-consensusstatement-on-covid-19-7-december-2021\#omicron-variant.

4 Sky News. Twitter. Dec 2021. https://twitter.com/SkyNews/status/1468842411291992067.

5 World Health Organization Office for Africa. Omicron spreads but severe cases remain low in South Africa. Dec 2021. https://www.afro.who.int/news/omicron-spreads-severe-cases-remainlow-south-africa

6 Covid-19: Omicron variant may be "milder" but its infection rate could be "devastating," expert warns. Sky News. 9 Dec 2021. https://news.sky.com/story/covid-19-omicron-variant-may-bemilder-but-its-infection-rate-could-be-devastating-expert-warns-12490761.

7 Cele S, Jackson L, Khan K. SARS-CoV-2 omicron has extensive but incomplete escape of Pfizer BNT162b2 elicited neutralization and requires ACE2 for infection.https://www.ahri.org/wp-content/uploads/2021/12/MEDRXIV-2021-267417v1-Sigal.pdfdoi: 10.1101/2021.12.08.21267417.

8 Pfizer and BioNTech provide update on omicron variant. Pfizer. 8 Dec 2021. https://www.pfizer.com/news/press-release/press-release-detail/pfizer-and-biontech-provide-update-omicronvariant.

9 Moderna exec says company could have omicron booster ready in March. Reuters. Dec 2021. https://www.reuters.com/business/healthcare-pharmaceuticals/moderna-exec-says-companycould-have-omicron-booster-ready-march-2021-12-01.

10 Mahase E. Covid-19: UK approves monoclonal antibody sotrovimab for over 12 s at high risk. BMJ 2021;375:n2990. doi: 10.1136/bmj.n2990 pmid: 34857518

11 Regeneron evaluating Regen-Cov and next generation antibodies against new omicron covid-19 variant. https://investor.regeneron.com/static-files/969bdb0b-53f5-46c7-94fb-7473ee7f5be3.

12 Mahase E. Covid-19: UK becomes first country to authorise antiviral molnupiravir. BMJ 2021;375:n2697. doi: 10.1136/bmj.n2697 pmid: 34737216

13 Mahase E. Covid-19: What have we learnt about the new variant in the UK?BMJ2020;371:m4944 doi: 10.1136/bmj.m4944 pmid: 33361120

14 Adesina A. Twitter. 5 Dec 2021. https://twitter.com/akin_adesina/status/1467634001934487559.

15 Torjesen I. Covid restrictions tighten as omicron cases double every two to three days. BMJ 2021;375doi: 10.1136/bmj.n3051.

This article is made freely available for use in accordance with BMJ's website terms and conditions for the duration of the covid-19 pandemic or until otherwise determined by BMJ. You may use, download and print the article for any lawful, non-commercial purpose (including text and data mining) provided that all copyright notices and trade marks are retained. 